\title{
The low Sr/Ba ratio on some extremely metal-poor stars ${ }^{\star}, \star \star$
}

\author{
M. Spite ${ }^{1}$, F. Spite ${ }^{1}$, P. Bonifacio ${ }^{1}$, E. Caffau ${ }^{1,2, \star \star \star}$, P. François ${ }^{1,3}$, and L. Sbordone $e^{4,2,1}$ \\ 1 GEPI Observatoire de Paris, CNRS, Université Paris Diderot, 92195 Meudon Cedex, France \\ e-mail: monique. spite@obspm. fr \\ 2 Zentrum für Astronomie der Universität Heidelberg, Landessternwarte, Königstuhl 12, 69117 Heidelberg, Germany \\ 3 Université de Picardie Jules Verne, 33 rue St-Leu, 80080 Amiens, France \\ ${ }^{4}$ Millennium Institute of Astrophysics, Universidad Catolica de Chile, Av. Vicuña Mackenna 4860, 782-0436 Macul, Santiago, Chile
}

Received 17 February 2014 / Accepted 2 September 2014

\begin{abstract}
Context. It has been noted that, in classical extremely metal-poor (EMP) stars, the abundance ratio of two well-observed neutroncapture elements, $\mathrm{Sr}$ and $\mathrm{Ba}$, is always higher than $[\mathrm{Sr} / \mathrm{Ba}]=-0.5$, which is the value of the solar r-only process; however, a handful of EMP stars have recently been found with a very low $\mathrm{Sr} / \mathrm{Ba}$ ratio.

Aims. We try to understand the origin of this anomaly by comparing the abundance pattern of the elements in these stars and in the classical EMP stars.

Methods. For a rigorous comparison with previous data, four stars with very low $\mathrm{Sr} / \mathrm{Ba}$ ratios were observed and analyzed in the same way as in the First Stars program: analysis within LTE approximation through 1D (hydrostatic) model atmosphere, providing homogeneous abundances of nine neutron-capture elements.

Results. In CS 22950-173, the only turnoff star of the sample, the $\mathrm{Sr} / \mathrm{Ba}$ ratio is, in fact, found to be higher than the r-only solar ratio, so the star is discarded. The remaining stars (CS 29493-090, CS 30322-023, HE 305-4520) are cool evolved giants. They do not present a clear carbon enrichment, but in evolved giants $\mathrm{C}$ is partly burned into $\mathrm{N}$, and owing to their high $\mathrm{N}$ abundance, they could still have initially been carbon-rich EMP stars (CEMP). The abundances of $\mathrm{Na}$ to $\mathrm{Mg}$ present similar anomalies to those in CEMP stars. The abundance patterns of the neutron-capture elements in the three stars are strikingly similar to a theoretical s-process pattern. This pattern could at first be attributed to pollution by a nearby AGB, but none of the stars presents a clear variation in the radial velocity indicating the presence of a companion. The stellar parameters seem to exclude any internal pollution in a TP-AGB phase for at least two of these stars. The possibility that the stars are early-AGB stars polluted during the core He flash does not seem compatible with the theory.
\end{abstract}

Key words. stars: abundances - stars: Population II - Galaxy: evolution - Galaxy: halo

\section{Introduction}

The heavy elements (heavier than $\mathrm{Zn}$ ) are thought to be built by two main processes. The s-process operates by slow neutron capture on seed nuclei on a long time scale and the capture is slow compared to the $\beta$ decay of the affected nucleus. It often happens in relatively low-mass stars at the end of their long evolution in their asymptotic giant branch (AGB) phase, (for example Käppeler et al. 2011; Bisterzo et al. 2012).

The r-process instead occurs on a very short time scale in violent events not yet clearly identified (e.g. Langanke \& Thielemann 2013; Aoki et al. 2013): explosions following the core collapse of massive supernovae, and/or mergings of neutron stars or of black holes, and/or jets, and/or gamma ray bursts, etc. These two distinct processes build generally different isotopes of a given heavy element, and different element ratios.

* Based on observations obtained with the ESO Very Large Telescope at Paranal Observatory, Chile (ID 077.D-0299(A) PI Bonifacio, and ID 078.B-0238(A) PI Spite), and on archive data ID 076.D-0451(A) PI Johnson.

$\star \star$ The line list and the abundances line by line are only available at the CDS via anonymous ftp to

cdsarc.u-strasbg.fr (130.79.128.5) or via

http://cdsarc.u-strasbg.fr/viz-bin/qcat?]/A+A/571/A40

$\star \star \star$ MERAC fellow.
Since the low-mass stars have a very long lifetime, the matter in the very first phases of the Galaxy could not be enriched by the late production of these low-mass stars in their AGB phase, therefore the heavy elements abundances in this matter must only reflect the r-process production.

The stars formed from this primitive matter are considered as key objects for constraining the r-process. These stars are extremely metal-poor, since at their birth the matter was enriched by a very small number of supernovae. Following Beers \& Christlieb (2005), stars are called extremely metal-poor (EMP), when $[\mathrm{Fe} / \mathrm{H}]^{1} \leq-3$. Here, for convenience, we extend this definition to stars with $[\mathrm{Fe} / \mathrm{H}] \leq-2.6$, although most of the stars considered are strictly EMP following the classification of Beers \& Christlieb (2005). We will consider two classes of EMP stars: the classical EMP stars (which are not carbon-rich) and the carbon-rich EMP stars (CEMP).

- The EMP stars. In the classical EMP stars, the scatter of the enrichment in neutron-capture elements is very large, some are rich in neutron-capture elements like CS 31082-001 (Hill et al. 2002; Siqueira Mello et al. 2013) with a mean enhancement reaching $[\mathrm{r} / \mathrm{Fe}] \simeq+1.0 \mathrm{dex}$, but others are poor with $[\mathrm{r} / \mathrm{Fe}] \simeq-1.0$ dex like HD 122563 (Honda et al. 2006). Generally, $[\mathrm{Eu} / \mathrm{Fe}]$ is used to measure the r-process enrichment. In the EMP stars it is not always easy to measure the abundance

1 In the classical notation, $[X / \mathrm{H}]=\log \left(N_{X} / N_{\mathrm{H}}\right)_{\text {star }}-\log \left(N_{X} / N_{\mathrm{H}}\right)_{\mathrm{Sun}}$. 
of Eu, but at these low metallicities there is a very good correlation between $[\mathrm{Eu} / \mathrm{Fe}]$ and $[\mathrm{Ba} / \mathrm{Fe}]$ (see, e.g., Mashonkina et al. 2010; Spite \& Spite 2014), and thus [Ba/Fe] can be used to estimate the r-process enrichment.

In Fig. $1[\mathrm{Sr} / \mathrm{Ba}]$ is plotted vs. [Ba/Fe] for the sample of EMP stars observed in the framework of the ESO Large Program First Stars, hereafter ESO-First Stars, following Hill et al. (2002), François et al. (2007), and Bonifacio et al. (2009). All the EMP stars are located in the upper lefthand corner of the figure, and the scatter of the ratio $[\mathrm{Sr} / \mathrm{Ba}]$ increases strongly when $[\mathrm{Ba} / \mathrm{Fe}]$ decreases. All the EMP stars have

$-[\mathrm{Ba} / \mathrm{Fe}] \lessgtr+1.0$,

$-[\mathrm{Sr} / \mathrm{Ba}]<0.5-[\mathrm{Ba} / \mathrm{Fe}]$

$-[\mathrm{Sr} / \mathrm{Ba}] \gtrsim-0.5$, and thus greater than the r-only solar value of this ratio: $[\mathrm{Sr} / \mathrm{Ba}]_{\mathrm{r}}=-0.5$ following Mashonkina \& Gehren (2001) and Mashonkina \& Christlieb (2014).

It has been shown that, generally speaking, in the EMP stars, the abundance pattern of the neutron-capture elements is not the same when $[\mathrm{Sr} / \mathrm{Ba}$ ] is high (as in CS 31082-001) and when [Sr/Ba] is low as in HD 122563 (Honda et al. 2006, 2007). It appears also that there are not two distinct populations of EMP stars r-rich and r-poor, but a continuous evolution of the abundance patterns of the neutron-capture elements with $[\mathrm{Ba} / \mathrm{Fe}]$ (see also Roederer et al. 2010).

- The CEMP stars. A significant fraction of the EMP stars is carbon-rich. Beers \& Christlieb (2005) consider that a star is carbon-rich when $[\mathrm{C} / \mathrm{Fe}] \geq+1.0$. As a comparison, following Bonifacio et al. (2009), the mean value of $[\mathrm{C} / \mathrm{Fe}]$ in normal EMP stars (not carbon-rich) is about $+0.40 \pm 0.2$. The fraction of carbon-enhanced stars increases when the metallicity decreases (see e.g. Lucatello et al. 2005; Carollo et al. 2014). They represent $20 \%$ of the stars at $[\mathrm{Fe} / \mathrm{H}]=-2$, and of the six stars known with $[\mathrm{Fe} / \mathrm{H}] \leq-4.5$, only one (Caffau et al. 2011, 2012) does not present a carbon enhancement. In Fig. 1, a set of CEMP stars has been gathered from LTE analyses of Aoki et al. (2001, 2002), Barbuy et al. (2005), Sivarani et al. (2006), Behara et al. (2010), Spite et al. (2013) and Yong et al. (2013).

Several CEMP stars are located in the same region as the EMP stars. They seem to have the same abundance pattern of neutron-capture elements as the EMP stars (Spite \& Spite 2014), and are generally not binaries (Starkenburg et al. 2014). They are classified CEMP-no (see also Carollo et al. 2014).

Other CEMP stars are strongly enriched in neutron-capture elements compared to the EMP stars. In Fig. 1 they have $[\mathrm{Ba} / \mathrm{Fe}] \gtrsim 1.0$ and $[\mathrm{Sr} / \mathrm{Ba}]<-0.5$. It has been shown that these stars have a high value of the ratio $[\mathrm{Pb} / \mathrm{Eu}]$ : a "s-process" signature (see, e.g., Mashonkina et al. 2012; Roederer et al. 2010; Sivarani et al. 2004). Such a signature may seem unexpected in stars formed so early in the Galaxy. But most of these stars have been found to have a variable radial velocity, indicating pollution by a nearby companion. In fact, the neutron-capture elements observed in these CEMP stars would not reflect the primitive matter that formed the star: they have been produced by the s-process in the higher mass companion of the binary in its AGB phase, and later transferred to the atmosphere of the (today observed) lower mass companion (see e.g. Izzard et al. 2009). In this paper, we will call these stars CEMP-s, without attempting to resolve the CEMP-rs subclass (where Eu is also enhanced, see, e.g., Barbuy et al. 2005) from a CEMP-s subclass (where it is not).

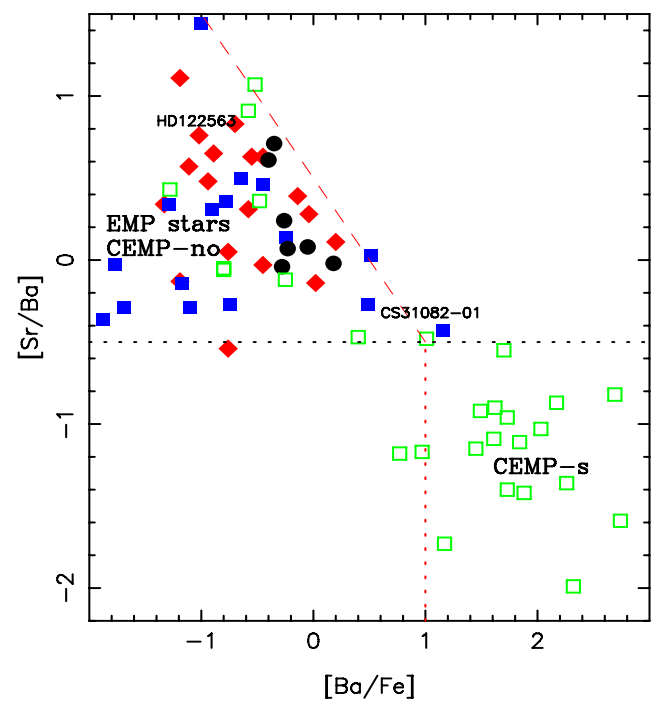

Fig. 1. $[\mathrm{Sr} / \mathrm{Ba}]$ vs. $[\mathrm{Ba} / \mathrm{Fe}]$ for i) our sample of EMP stars from the Large Program First Stars: filled symbols (blue squares for unmixed giants, Spite et al. 2005, red diamonds for mixed giants, black circles for dwarfs); and ii) green open squares for the CEMP stars in the same range of metallicity gathered from Aoki et al. (2001, 2002), Barbuy et al. (2005), Sivarani et al. (2006), Behara et al. (2010), Spite et al. (2013), and Yong et al. (2013). The dotted line at $[\mathrm{Sr} / \mathrm{Ba}]=-0.5$ represents the value of the r-only solar value of this ratio (Mashonkina \& Gehren 2001). The EMP stars are all located in the left upper corner of the figure, many CEMP stars are located in the same region (they are CEMP-no), but another group (CEMP-s) has a high abundance of Ba: $[\mathrm{Ba} / \mathrm{Fe}] \gtrsim+1$ associated with a low value of $[\mathrm{Sr} / \mathrm{Ba}$ ] (lower right region). In the left upper corner, the stars are gathered below the dashed line: $[\mathrm{Sr} / \mathrm{Ba}]=0.5-[\mathrm{Ba} / \mathrm{Fe}]$. (Color figures are available in the electronic edition.)

Recently, Aoki et al. (2013) have extracted normal (not carbon-rich) EMP stars $([\mathrm{Fe} / \mathrm{H}] \leq-2.5)$ in the SAGA Database (Suda et al. 2008) with measured values of Sr and Ba (260 stars). Almost all these stars present a ratio $[\mathrm{Sr} / \mathrm{Ba}]>-0.5$, as expected from Fig. 1 for normal EMP stars. However they point out that among these stars, a subsample of six EMP stars (not C-rich) had a surprisingly low-[Sr/Ba] ratio, lower than the r-only solar value of this ratio $([\mathrm{Sr} / \mathrm{Ba}]=-0.5)$. In these stars the $[\mathrm{Sr} / \mathrm{Ba}]$ ratio is similar to the ratio observed in the CEMP-s stars, but they have a ratio $[\mathrm{Ba} / \mathrm{Fe}]<+1$. This paper is an attempt to better understand the cause of these anomalies.

\section{Observations of the star sample}

In the subsample of Aoki et al. (2013), two stars are suspected of being binaries. A pollution by a companion could thus be suspected of being responsible for their abundance anomalies. But four EMP stars are found to have a ratio $[\mathrm{Sr} / \mathrm{Ba}] \ll-0.5$ and $[\mathrm{Ba} / \mathrm{Fe}]<+1$ and are, to date, not suspected of being binaries. These stars are presented in Table 1.

The star CS 30322-023 has been studied in detail by Masseron et al. (2006, 2010) and Aoki et al. (2007). We analyzed high-resolution spectra for the three other stars. The spectra of CS 22950-173 and HE 0305-4520 were obtained at the VLT with the UVES spectrograph (Dekker et al. 2000) in the course of our own observing programs. The UVES spectra of CS 29493-090 (HE 2156-3130) were retrieved from the ESO archive.

The resolving power of the spectra is about 40000 with five pixels per resolution element. The spectra cover the ranges $330<\lambda<451 \mathrm{~nm}$ (blue arm) and $480<\lambda<680 \mathrm{~nm}$ 
Table 1. Stars selected by Aoki et al. (2013) with $[\mathrm{Fe} / \mathrm{H}] \leq-2.5$, $[\mathrm{Ba} / \mathrm{Fe}]<1.0$ and $[\mathrm{Sr} / \mathrm{Ba}] \ll-0.5$.

\begin{tabular}{|c|c|c|c|c|c|c|}
\hline Star & {$[\mathrm{Fe} / \mathrm{H}]$} & {$[\mathrm{Ba} / \mathrm{Fe}]$} & {$[\mathrm{Sr} / \mathrm{Ba}]$} & $T_{\text {eff }}$ & $\log g$ & Ref \\
\hline CS 22950-173 & -2.50 & -0.04 & -0.72 & 6800 & 4.5 & 1 \\
\hline CS 29493-090 & -2.82 & +0.52 & -1.41 & 4700 & 1.3 & 2 \\
\hline CS 30322-023 & -3.40 & +0.54 & -1.05 & 4100 & -0.3 & 3 \\
\hline " & -3.26 & +0.59 & -1.10 & 4300 & 1.0 & 4 \\
\hline HE $0305-4520$ & -2.91 & +0.59 & -1.25 & 4820 & 1.3 & 2 \\
\hline
\end{tabular}

References. 1) Preston \& Sneden (2000); 2) Barklem et al. (2005); 3) Masseron et al. (2006); 4) Aoki et al. (2007).

Table 2. Radial velocities (geocentric and barycentric) of the three stars studied.

\begin{tabular}{lcrrr}
\hline \hline Star & MJD & $\begin{array}{r}R V \\
\text { geo })\end{array}$ & $\begin{array}{r}R V \\
\text { corr }\end{array}$ & $\begin{array}{r}R V \\
\text { (bary) }\end{array}$ \\
\hline CS 22950-173 & 53848.34 & +38.7 & +29.8 & +68.5 \\
& & & & \\
CS 29493-090 & 53637.17 & +287.9 & -18.8 & +269.1 \\
CS 29493-090 & 53645.18 & +291.5 & -21.7 & +269.8 \\
CS 29493-090 & 53645.22 & +291.85 & -21.75 & +270.1 \\
& & & & \\
HE 0305-4520 & 54024.25 & +137.2 & -0.6 & +136.6 \\
\hline
\end{tabular}

Notes. The precision of the radial velocities is about $1 \mathrm{~km} \mathrm{~s}^{-1}$.

(red arm). The spectra were reduced (optimum extraction, division by the flat field, wavelength calibration) using the standard UVES pipeline. The $\mathrm{S} / \mathrm{N}$ of the spectra (per pixel) at $420 \mathrm{~nm}$ is 110 for CS 22950-173, 80 for CS 29493-090 and 100 for HE 0305-4520.

Since the existence or non-existence of a companion is essential for explaining the peculiar heavy element patterns in metal-poor stars, we carefully measured the radial velocity of the three new low-Sr/Ba stars (Table 2). The measurements were done on the blue spectra, and the precision of the measurements is about $1 \mathrm{~km} \mathrm{~s}^{-1}$. These measurements are discussed in Sects. 4 and 6.1.1 and compared to the values found in the literature.

\section{Analysis}

We carried out a classical LTE analysis, which is homogeneous with the analysis of the ESO-First Stars sample: red giant branch (RGB) stars (Cayrel et al. 2004) and turnoff stars (Bonifacio et al. 2009), using OSMARCS model atmospheres (Gustafsson et al. 1975, 2003) and the turbospectrum spectral synthesis code (Alvarez \& Plez 1998; Plez 2012).

For the two giants, the effective temperatures $T_{\text {eff }}$ were taken from Barklem et al. (2005) and deduced from colors. For the turnoff star CS 22950-173, we adopted a temperature based on 3D profiles of the $\mathrm{H} \alpha$ wings taking into account the influence of the gravity on this profile iteratively (Sbordone et al., in prep.). This temperature is a little higher than the value adopted by Sbordone et al. (2010) but a little lower than the temperature adopted by Preston \& Sneden (2000).

The microturbulence velocity was derived from the Fe I lines, requiring that the abundance derived for individual lines be independent of the equivalent width of the line, and the surface gravity $\log g$ was determined by requiring that the $\mathrm{Fe}$ and $\mathrm{Ti}$ abundances derived from neutral and ionized lines be the same.
Table 3. Parameters of the models adopted for the three stars analyzed here and the corresponding errors.

\begin{tabular}{lcrcc}
\hline \hline Star & $T_{\text {eff }}$ & $\log g$ & $v_{\mathrm{t}}$ & {$[\mathrm{Fe} / \mathrm{H}]$} \\
\hline CS 22950-173 & 6615 & 4.1 & 1.4 & -2.6 \\
CS 29493-090 & 4700 & 1.3 & 1.9 & -3.1 \\
HE 0305-4520 & 4820 & 1.3 & 2.0 & -3.0 \\
\hline
\end{tabular}

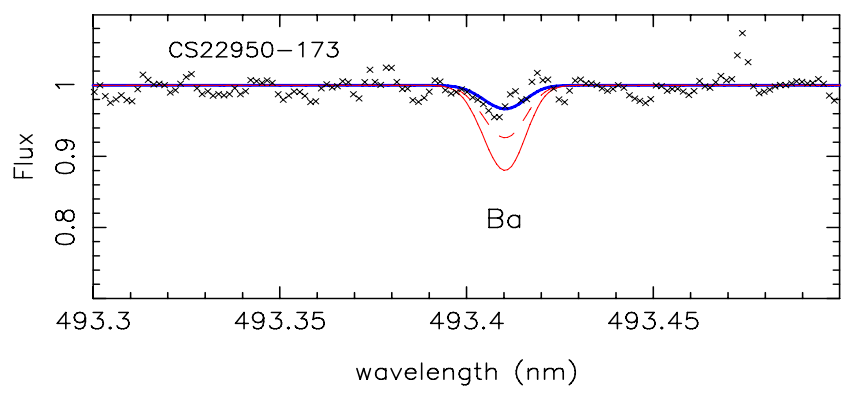

Fig. 2. Profile of the 493.4 Ba II lines in CS 22950-173 computed with our adopted model (Table 1 ) and $[\mathrm{Ba} / \mathrm{Fe}]=-0.53$ (adopted value, thick blue line), and $[\mathrm{Ba} / \mathrm{Fe}]=-0.04$ (thin red line). The dashed line corresponds to a profile of the Ba line computed with the model adopted by Preston \& Sneden (2000) and $[\mathrm{Ba} / \mathrm{Fe}]=-0.04$.

The adopted parameters are given in Table 3. These parameters are in the same range as the models adopted for the ESO-First Stars sample, giant and turnoff stars (Cayrel et al. 2004; Bonifacio et al. 2009) and lead to the same uncertainties: about $100 \mathrm{~K}$ on $T_{\text {eff }}, 0.2$ dex on $\log g$, and $0.2 \mathrm{~km} \mathrm{~s}^{-1}$ on $v_{\mathrm{t}}$, which induces an uncertainty of about $0.1 \mathrm{dex}$ on $[\mathrm{Fe} / \mathrm{H}]$.

\section{The turnoff star CS 22950-173}

In our set of stars, CS 22950-173 is the only turnoff star, and the chemical composition of the atmosphere of this star could not be affected by a mixing with deep layers. Following Aoki et al. (2013), this star has been found to be a binary by Preston \& Sneden (2000), suggesting that an anomaly in its abundance pattern could be due to a mass transfer from a now defunct companion, when it was in its AGB phase. However, we checked that Preston \& Sneden (2000) do not indicate in their Table 1 that this star is suspected to be a binary, and it is even listed (their Table 4) among the "blue metal-poor stars with constant radial velocities". Moreover, in our spectrum obtained in 2006, we measured a barycentric radial velocity (Table 2 ), in good agreement with the value $\left(=69.4 \pm 1 \mathrm{~km} \mathrm{~s}^{-1}\right)$ measured by Preston \& Sneden (2000) on spectra obtained between 1993 and 1998.

A first analysis of this star has been published in Sbordone et al. (2010) giving the abundances of Fe and Li. A new more complete analysis will be published soon by Sbordone et al. (in prep.). The $\mathrm{CH}$ band is very weak, and we found $[\mathrm{C} / \mathrm{Fe}] \leq$ +0.78: the star is not a CEMP star, according to the definition of Beers \& Christlieb (2005).

We could measure two Sr II lines at 407.771 and $421.552 \mathrm{~nm}$ and two Ba II lines at 493.408 and $614.171 \mathrm{~nm}$, and we obtained $[\mathrm{Sr} / \mathrm{H}]=-3.24$ and $[\mathrm{Ba} / \mathrm{H}]=-3.13([\mathrm{Ba} / \mathrm{Fe}]=-0.53)$ i.e. $[\mathrm{Sr} / \mathrm{Ba}]=-0.11$.

In Fig. 2 we compare the observed spectrum in the region of the $\mathrm{Ba}$ line at $493.408 \mathrm{~nm}$ with

(i) synthetic profiles computed with our adopted model and $[\mathrm{Ba} / \mathrm{Fe}]=-0.53$ (adopted value) and $[\mathrm{Ba} / \mathrm{Fe}]=-0.04$ (Preston \& Sneden value), and 
Table 4. LTE abundances in low- $[\mathrm{Sr} / \mathrm{Ba}]$ ratio giant stars. For $\mathrm{Na}$ we give also the NLTE value.

\begin{tabular}{|c|c|c|c|c|c|c|}
\hline & \multicolumn{2}{|c|}{ CS 30322-023 } & \multicolumn{2}{|c|}{ CS 29493-090 } & \multicolumn{2}{|c|}{ HE $0305-4520$} \\
\hline$[\mathrm{Fe} / \mathrm{H}]$ & \multicolumn{2}{|c|}{-3.40} & \multicolumn{2}{|c|}{-3.10} & \multicolumn{2}{|c|}{-2.95} \\
\hline${ }^{12} \mathrm{C} /{ }^{13} \mathrm{C}$ & & & 5. & & 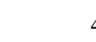 & \\
\hline & {$[X / \mathrm{Fe}]$} & $\bar{\sigma}$ & {$[X / \mathrm{Fe}]$} & $\sigma$ & {$[X / \mathrm{Fe}]$} & $U$ \\
\hline $\mathrm{C}_{(\mathrm{CH})}$ & 0.80 & - & 0.73 & 0.10 & 0.42 & 0.10 \\
\hline $\mathrm{N}$ & 2.91 & - & 1.51 & .15 & 1.58 & 0.15 \\
\hline $\mathrm{C}+\mathrm{N}$ & 1.70 & - & 1.03 & - & 0.99 & - \\
\hline $\mathrm{O}$ & 0.63 & - & - & - & 0.83 & 0.10 \\
\hline $\mathrm{Na}$ & 1.29 & 0.24 & 1.13 & 0.10 & 1.04 & 0.10 \\
\hline $\mathrm{Na}_{(\mathrm{NLTE})}$ & 0.79 & 0.24 & 0.53 & 0.10 & 0.54 & 0.10 \\
\hline $\mathrm{Mg}$ & 0.80 & 0.10 & 0.98 & 0.13 & 0.36 & 0.11 \\
\hline $\mathrm{Ca}$ & 0.30 & 0.12 & 0.27 & 0.10 & 0.26 & 0.10 \\
\hline Ti I & -0.20 & 0.19 & 0.11 & 0.05 & 0.16 & 0.05 \\
\hline Ti II & 0.23 & 0.13 & 0.17 & 0.05 & 0.21 & 0.05 \\
\hline $\mathrm{Fe} \mathrm{I}$ & 0.01 & 0.18 & -0.04 & 0.05 & -0.01 & 0.05 \\
\hline Fe II & -0.01 & 0.19 & 0.03 & 0.05 & 0.04 & 0.05 \\
\hline $\mathrm{Sr}$ & -0.50 & - & -1.02 & 0.11 & -0.76 & 0.11 \\
\hline $\mathrm{Y}$ & -0.36 & 0.15 & -0.82 & 0.10 & -0.58 & 0.10 \\
\hline $\mathrm{Zr}$ & 0.14 & & -0.50 & 10 & -0.38 & 0.11 \\
\hline $\mathrm{Ba}$ & 0.52 & 0.1 & 0.43 & 0.10 & 0.32 & 0.10 \\
\hline $\mathrm{La}$ & 0.46 & 0.1 & 0.23 & 0.12 & 0.09 & 0.12 \\
\hline $\mathrm{Ce}$ & 0.59 & 0.24 & 0.19 & 0.10 & 0.31 & 0.12 \\
\hline $\mathrm{Nd}$ & 0.57 & 0.29 & 0.23 & 0.11 & 0.32 & 0.10 \\
\hline $\mathrm{Eu}$ & -0.63 & - & -0.21 & 0.11 & -0.31 & 0.11 \\
\hline Dy & -0.13 & - & - & - & - & - \\
\hline $\mathrm{Pb}$ & 1.49 & 0.20 & 1.15 & 0.18 & 1.25 & 0.22 \\
\hline
\end{tabular}

Notes. The data for CS 30322-023 are taken from Masseron et al. (2006).

(ii) the synthetic profile computed with the model of Preston \& Sneden (2000) and $[\mathrm{Ba} / \mathrm{Fe}]=-0.04$.

It can be deduced from this figure that the value of $[\mathrm{Ba} / \mathrm{Fe}]$ measured by Preston \& Sneden is not compatible with our spectrum and the model we adopted. If the model of Preston \& Sneden (2000) had been adopted, a higher value of $[\mathrm{Ba} / \mathrm{Fe}]$ would be found $([\mathrm{Ba} / \mathrm{Fe}] \simeq-0.35)$ but also a higher value of $[\mathrm{Sr} / \mathrm{Fe}]$ and thus finally, the ratio $[\mathrm{Sr} / \mathrm{Ba}]$ would remain unchanged. Finally, the star CS 22950-173, with [Sr/Ba] $\simeq-0.1$, is not a low-Sr/Ba star, and we will no more consider this star in the following.

\section{The giant stars}

The abundances of the elements in the three low-Sr/Ba giants, CS 29493-090, HE 0305-4520 (this paper), and CS 30322-023 (Masseron et al. 2006, 2010) are given in Table 4 with the stochastic errors arising from random uncertainties in the oscillator strengths and in the measurement of the equivalent widths or the profiles. Systematic uncertainties are mainly due to the adopted stellar parameters. The total uncertainty can be estimated as the quadratic sum of the stochastic and the systematic errors. Because of the similarity of the response of a given set of elements to changes in stellar parameters, systematic errors largely cancel out, reducing the uncertainty on the relative abundances. In the range of temperature gravity and metallicity considered here, the systematic error on $[X / \mathrm{Fe}]$ is less than 0.1 (see Hill et al. 2002; Cayrel et al. 2004; François et al. 2007).

It is interesting to note that in CS 29493-090 (Table 4) the abundance of some elements (C, N, Na, Mg, Ba, Fe) has been determined independently by Masseron et al. (2012). They used a model that is only slightly different $\left(T_{\text {eff }}=4700 \mathrm{~K}, \log g=0.9\right.$, $v_{\mathrm{t}}=1.6 \mathrm{~km} \mathrm{~s}^{-1}$ ), but the agreement in the determination of $[\mathrm{Fe} / \mathrm{H}]$ and $[X / \mathrm{Fe}]$ is good with a $\Delta[X / \mathrm{Fe}]<0.15 \mathrm{dex}$. The main difference is observed for $[\mathrm{N} / \mathrm{Fe}]$ because Masseron et al. only used the $\mathrm{CN}$ band, while we used the $\mathrm{CN}$ and the $\mathrm{NH}$ bands. If we had only used the $\mathrm{CN}$ band, we would have found $[\mathrm{N} / \mathrm{Fe}]=+1.34$, and the difference $\Delta[\mathrm{N} / \mathrm{Fe}]$ would drop down to 0.11 dex. The line list, and the abundances line by line are available in electronic form at the CDS.

Below we compare the abundances of the elements in these three stars,

(i) to our set of classical EMP stars studied in the same way in the frame of the ESO-First Stars program (Hill et al. 2002; Cayrel et al. 2004; Spite et al. 2005; Bonifacio et al. 2009),

(ii) to CEMP stars, observed and analysed also homogeneously in the frame of the ESO-First Stars program: CS 22892-052 (Sneden et al. 2003; Cayrel et al. 2004), CS 22949-037 (McWilliam et al. 1995; Depagne et al. 2002), and CS 31080-095, CS 22958-045, CS 29528-041 (Sivarani et al. 2006), and to CEMP stars in the same range of metallicity, gathered from the literature Aoki et al. (2001, 2002), Barbuy et al. (2005), Behara et al. (2010), Spite et al. (2013), Yong et al. (2013). We have divided the CEMP stars in two subclasses: the CEMP-s, with $[\mathrm{Ba} / \mathrm{Fe}]>1.0$ and the CEMP-no with $[\mathrm{Ba} / \mathrm{Fe}] \leq 1.0$, (see Fig. 1$)$.

\subsection{CNO abundances}

With a ratio $[\mathrm{C} / \mathrm{Fe}]$ between 0.42 and 0.80 dex (Table 4), the three low $\mathrm{Sr} / \mathrm{Ba}$ stars are not CEMP stars according to the definition of Beers \& Christlieb (2005): $[\mathrm{C} / \mathrm{Fe}]>+1$.

But in the atmosphere of giant stars, AGB, or even red giant branch (RGB) stars, the carbon abundance does not always represent the abundance of $\mathrm{C}$ in the gas that formed the star. It has been shown, in particular, that in classical EMP stars evolving along the RGB, an extra mixing occurs when a star crosses the bump in the luminosity function (see: Spite et al. 2005, 2006). At $[\mathrm{Fe} / \mathrm{H}] \approx-3.0$, the extra mixing occurs for a temperature of about $4800 \mathrm{~K}$ and a (spectroscopic) gravity $\approx 1.5$. The atmosphere is mixed with $\mathrm{CNO}$ processed material: $[\mathrm{C} / \mathrm{Fe}]$ decreases and $[\mathrm{N} / \mathrm{Fe}]$ increases, but $[(\mathrm{C}+\mathrm{N}) / \mathrm{Fe}]$ remains (almost) constant with a mean value close to +0.3 dex (see Fig. 3 ).

The three low-Sr/Ba stars are cool giants (Table 3 ), and thus it is possible that in their atmosphere, part of the original carbon has been transformed into nitrogen. As a consequence, it is more suitable to compare the abundance of $\mathrm{C}+\mathrm{N}$ in the low$\mathrm{Sr} / \mathrm{Ba}$ stars to the abundance of $\mathrm{C}+\mathrm{N}$ in classical EMP stars and in CEMP stars (Fig. 3).

As for the CEMP-s or the CEMP-no stars, the three low$\mathrm{Sr} / \mathrm{Ba}$ stars, present a ratio $[(\mathrm{C}+\mathrm{N}) / \mathrm{Fe}]$ higher than the classical EMP stars (Fig. 3). It is interesting to note that the CEMP-s and the CEMP-no stars occupy different regions of the figure, the CEMP-s having, at a given metallicity, a higher value of $[(\mathrm{C}+\mathrm{N}) / \mathrm{Fe}]$ than the CEMP-no. This difference could reflect the different origins of the overabundance of $\mathrm{C}$ and $\mathrm{N}$ in CEMP-s and CEMP-no stars (see also, Spite et al. 2013).

The three low-Sr/Ba stars are located in the region of the CEMP-no stars. They could have been born carbon-rich and later, could have undergone an extra mixing, which would explain their relatively low carbon abundance associated with a high value of the abundance of nitrogen. This extra mixing is also characterized by a low value of the ${ }^{12} \mathrm{C} /{ }^{13} \mathrm{C}$ ratio (close to the equilibrium value), which is indeed observed in these three stars (see upper part of Table 4). 
M. Spite: Sr poor extremely metal-poor stars

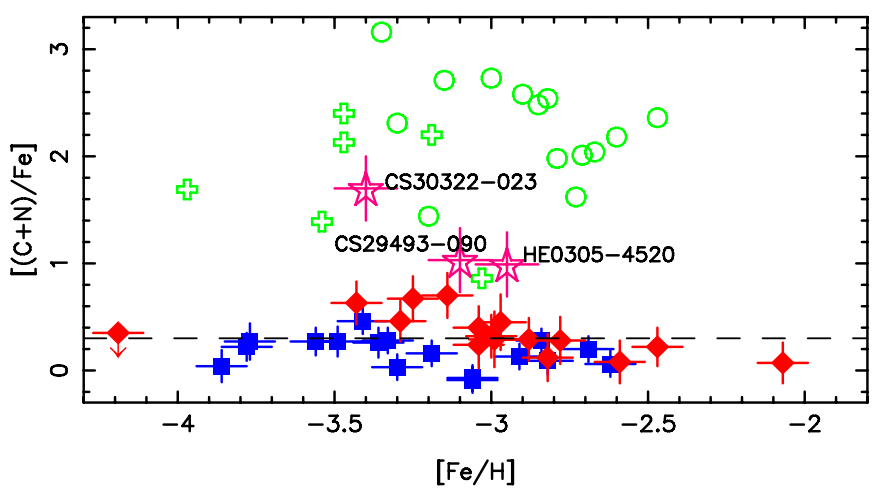

Fig. 3. Comparison of the abundance of $[(\mathrm{C}+\mathrm{N}) / \mathrm{Fe}]$. (i) For the sample of EMP stars studied in the framework of the Large Program First Stars: filled blue squares for unmixed RGB stars, filled red diamonds for mixed RGB's, and filled black circles for the turnoff stars (no EMP turnoff stars appear in this figure, because it was not possible to measure the nitrogen abundance in these stars Bonifacio et al. 2009); (ii) for CEMP-no (open green crosses); (iii) for CEMP-s (open green circles), and (iv) for the three low-[Sr/Ba] giants here studied (open pink star symbols). These three low-[Sr/Ba] stars are located in the region generally occupied by the carbon-rich stars.

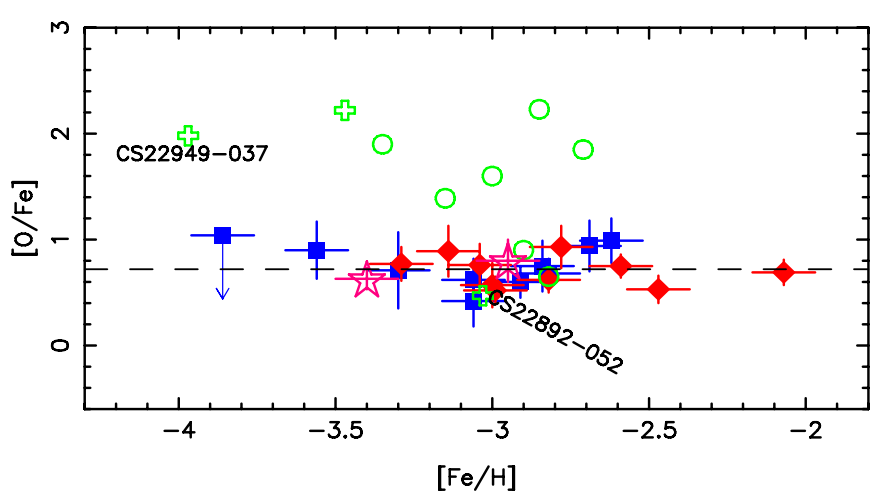

Fig. 4. Comparison of the abundance of oxygen in a sample of RGB stars studied in the framework of the ESO Large Program First Stars, in CEMP-s and CEMP-no stars and in the low-Sr/Ba giants. The symbols are the same as in Fig. 3. The three low-Sr/Ba giants have an oxygen abundance similar to the abundance measured in the classical EMP stars, but several CEMP stars also have a normal oxygen abundance.

In two of these stars the oxygen abundance could be derived from the forbidden line at $630 \mathrm{~nm}$, and when compared to the set of normal EMP stars this abundance appears to be normal, although in some carbon-rich stars (but not all), it is very high (see Fig. 4).

In Figs. 3 and 4, only the giants of the Large Program First Stars appear, since it has not been possible to measure $\mathrm{N}$ and $\mathrm{O}$ in the subsample of turnoff stars.

\subsection{The light elements from $\mathrm{Na}$ to $\mathrm{Mg}$}

Since in these very metal-poor stars, the sodium abundance is deduced from the Na I resonance lines, the NLTE correction can reach -0.5 dex, and it is different in turnoff and giant stars. As a consequence, in Table 4 and in Fig. 5, in order to allow a comparison with the Na abundance in the classical EMP and CEMP stars, the $[\mathrm{Na} / \mathrm{Fe}]_{\mathrm{NLTE}}$ has been estimated from the computations of Gehren et al. (2004) and Andrievsky et al. (2007).

In Figs. 5 and 6 we compare the sodium and magnesium abundances in classical EMP, CEMP-s, CEMP-no stars and in

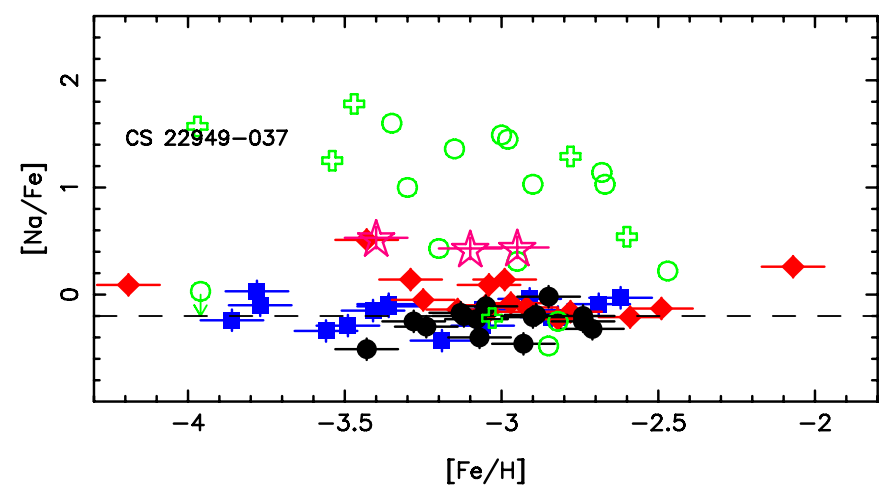

Fig. 5. Comparison of the abundance of sodium in a sample of RGB stars studied in the framework of the ESO Large Program First Stars and in the three low- $[\mathrm{Sr} / \mathrm{Ba}]$ giants studied here. The symbols are the same as in Fig. 3. The three low-Sr/Ba giants have a high sodium abundance like several RGB mixed stars, but $[\mathrm{Na} / \mathrm{Fe}]$ in these stars is less than the value observed in the majority of the CEMP-s or CEMP-no stars.

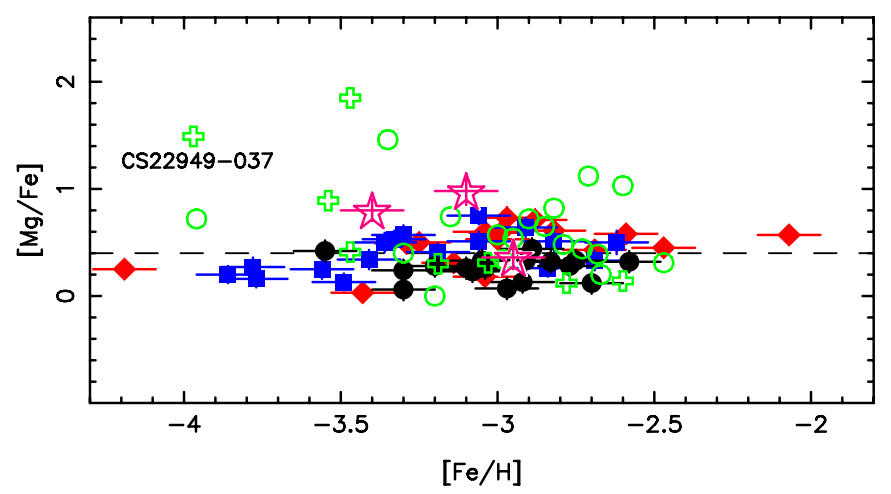

Fig. 6. Comparison of the abundance of magnesium in the ESO-First Stars and in the three low-Sr/Ba giants studied here. The symbols are the same as in Fig. 3. In two low- $\mathrm{Sr} / \mathrm{Ba}$ giants, $[\mathrm{Mg} / \mathrm{Fe}]$ is higher than in the classical EMP stars as in many CEMP-s or CEMP-no stars, but in the third one $[\mathrm{Mg} / \mathrm{Fe}]$ is close to the mean value, as it is also in many CEMP-s or CEMP-no stars.

the three peculiar low-Sr/Ba stars. The Na abundance in these three stars is high (as in several mixed RGB stars), but not as high as in most CEMP stars (CEMP-s or CEMP-no). The slight overabundance of magnesium in these stars is normal for EMP or CEMP stars, but $[\mathrm{Mg} / \mathrm{Fe}]$ is rather high in two of these stars (Fig. 6), but not as high as in many CEMP-s or CEMP-no stars.

The classical EMP stars present a very low scatter of $[\mathrm{C}+\mathrm{N} / \mathrm{Fe}],[\mathrm{O} / \mathrm{Fe}],[\mathrm{Na} / \mathrm{Fe}],[\mathrm{Mg} / \mathrm{Fe}]$. This low scatter is illustrated in Figs. 3-6 (filled symbols). The pattern of the abundances of the elements from C to Mg in CS 30322-023, CS 29493-090, and HE0305-4520 shows that these stars are not classical EMP stars and it allows us to classify them rather among the CEMP stars. The abundances of these light elements in the CEMP-s or the CEMP-no stars is so scattered that it is not possible at this stage to constrain the origin of the overabundance of $\mathrm{C}+\mathrm{N}, \mathrm{Na}$, and $\mathrm{Mg}$ better in the three low-Sr/Ba stars.

\subsection{The neutron-capture elements from $\mathrm{Sr}$ to $\mathrm{Pb}$}

To allow a more precise comparison of the low-Sr/Ba stars to the different subsamples of metal-poor stars (EMP stars, CEMP stars), in Fig. 7 we have plotted [Sr/Ba] vs. [Ba/Fe] for only giant stars within a narrow range of metallicity $[\mathrm{Fe} / \mathrm{H}]=-3.1 \pm$ 0.4 . These stars have model parameters very close to those of the 


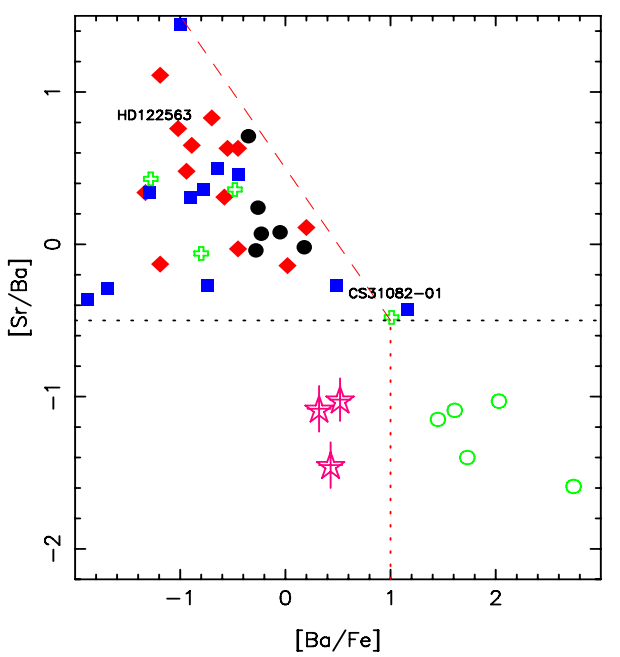

Fig. 7. $[\mathrm{Sr} / \mathrm{Ba}]$ vs. $[\mathrm{Ba} / \mathrm{Fe}]$ for i) our sample of EMP stars (Large Program First Stars restricted to the stars with $-3.5 \leq[\mathrm{Fe} / \mathrm{H}] \leq-2.7$ ), for ii) the CEMP stars in the same range of metallicity gathered from Aoki et al. (2001, 2002), Depagne et al. (2002), Barbuy et al. (2005), and Yong et al. (2013), and for iii) the three low-Sr/Ba giants (this paper). Symbols are the same as in Fig. 3. The three low-Sr/Ba giants occupy a very peculiar position in this diagram: they associate a low value of $[\mathrm{Sr} / \mathrm{Ba}$ ] like in the CEMP-s stars to a relatively low value of $[\mathrm{Ba} / \mathrm{Fe}]([\mathrm{Ba} / \mathrm{Fe}]<1)$ like in the classical EMP stars or the CEMP-no.

low-Sr/Ba stars, and as a consequence the comparison is even more suitable.

As can be seen, the three low-Sr/Ba stars occupy a very peculiar position in this diagram, and this position is not the consequence of a difference in metallicity since the different subsamples of stars in this figure have the same mean metallicity. The low-Sr/Ba stars are Ba-rich with $[\mathrm{Ba} / \mathrm{Fe}]>0.0$ like the EMP $\mathrm{r}$-rich stars, but their $\mathrm{Sr}$ abundance is low with $[\mathrm{Sr} / \mathrm{Fe}]<-0.5$, and as a consequence, the ratio $[\mathrm{Sr} / \mathrm{Ba}]$ is less than the lower limit for an r-process production: $[\mathrm{Sr} / \mathrm{Ba}]=-0.5$ (Mashonkina $\&$ Gehren 2001). The ratio [Sr/Ba] in the three low-Sr/Ba giants is similar to the one observed in the CEMP-s stars (Fig. 7) but with a lower $[\mathrm{Ba} / \mathrm{Fe}]$ ratio.

In panel A of Fig. 8, we compare the abundance ratios $[X / \mathrm{Fe}]$ (where $X$ is any of the neutron-capture elements) in the low$\mathrm{Sr} / \mathrm{Ba}$ stars (Table 4 ) to the ratios observed in r-rich stars, which are a good proxy for the typical r-process (Siqueira Mello et al. 2013; Plez et al. 2004). The behaviors are completely different, even the behavior of the $\mathrm{Sr}, \mathrm{Y}, \mathrm{Zr}$ group is different, but the main difference is in the $[\mathrm{Pb} / \mathrm{Eu}]$ ratio. This is a clear indication that in our low-Sr/Ba stars, the neutron-capture elements were not formed by the r-process.

In panel $\mathrm{B}$, we compare the abundance ratios $[X / \mathrm{Fe}]$ in the three low-Sr/Ba stars to the s-process production during thermal pulses in a moderately metal-poor AGB $(Z=0.001)$ following Goriely \& Mowlavi (2000). In this figure we shifted those computed $[X / \mathrm{Fe}]$ ratios by -1.4 dex for an easier comparison to the observations. In spite of the higher metallicity of the AGB model in the computations of Goriely \& Mowlavi (2000), the abundance pattern is quite similar.

The similarity between the s-process computations during thermal pulses of AGB stars (Goriely \& Mowlavi 2000) is striking (in particular the ratio $[\mathrm{Pb} / \mathrm{Eu}]$ ) and strongly support that the neutron-capture elements in the low-Sr/Ba stars have mainly been formed by the s-process.

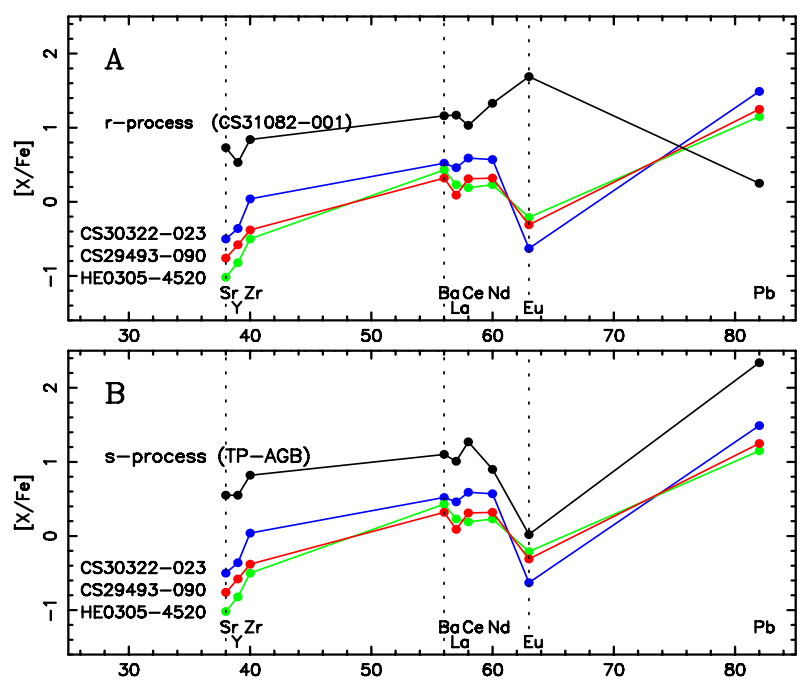

Fig. 8. Neutron-capture element ratios relative to iron $[\mathrm{X} / \mathrm{Fe}]$, in the three low-Sr/Ba stars (CS 30322-023, blue line; CS 29493-090, red line; HE 0305-4520, green line), are compared in A) to the ratios observed in CS 31082-001, a typical example of the r-process, in B) to the s-process computations in an AGB star during the thermal pulses shifted by -1.4 dex.

\section{Discussion}

\subsection{Were the low-Sr/Ba stars enriched by the ejecta of a extinct $A G B$ star?}

In panel A of Fig. 9, we compare the abundance pattern of two CEMP-s stars CS 22948-027 or CS 29497-034 (following Barbuy et al. 2005) to the abundance pattern of a classical r-rich EMP star CS 31082-001 (Siqueira Mello et al. 2013).

Compared to the abundance pattern of the r-elements in the Sun the CEMP-s stars present a strong overabundance of $\mathrm{Ba}, \mathrm{La}$, $\mathrm{Ce}, \mathrm{Nd}$, and $\mathrm{Pb}$ relative to $\mathrm{Eu}$.

In panel $\mathrm{B}$, we compare the abundance pattern of the three low-Sr/Ba stars to CS 31082-001 and the solar r-process abundance pattern. The low-Sr/Ba stars and the CEMP-s stars have similar characteristics: strong enhancement of $\mathrm{Ba}, \mathrm{La}, \mathrm{Ce}$, and $\mathrm{Pb}$ relative to $\mathrm{Eu}$ when compared to r-process rich stars and/or to the the solar r-process although the overabundances of the $\mathrm{Ba}$-group and of $\mathrm{Pb}$ (relative to $\mathrm{Eu}$ ) is more pronounced in the low-Sr/Ba stars.

The CEMP-s stars such as CS 22948-027 or CS 29497-034 are generally found to be binaries, and thus the distribution of their neutron-capture elements is explained by a mass transfer from an earlier AGB companion (now, a white dwarf), that has produced neutron-capture elements by the s-process. Would it be possible that the three low $\mathrm{Sr} / \mathrm{Ba}$ stars in Table 4 be RGB binaries that have been enriched by an AGB companion?

\subsubsection{Radial velocities of the three low-Sr/Ba stars}

The radial velocities of the three low-Sr/Ba stars have been carefully measured:

\section{- CS 30322-023}

The radial velocity of this star has been studied in detail by Masseron et al. (2006) from 12 spectra obtained between 2001 and 2006, and they conclude that there was no firm support for the binary nature of this star. 


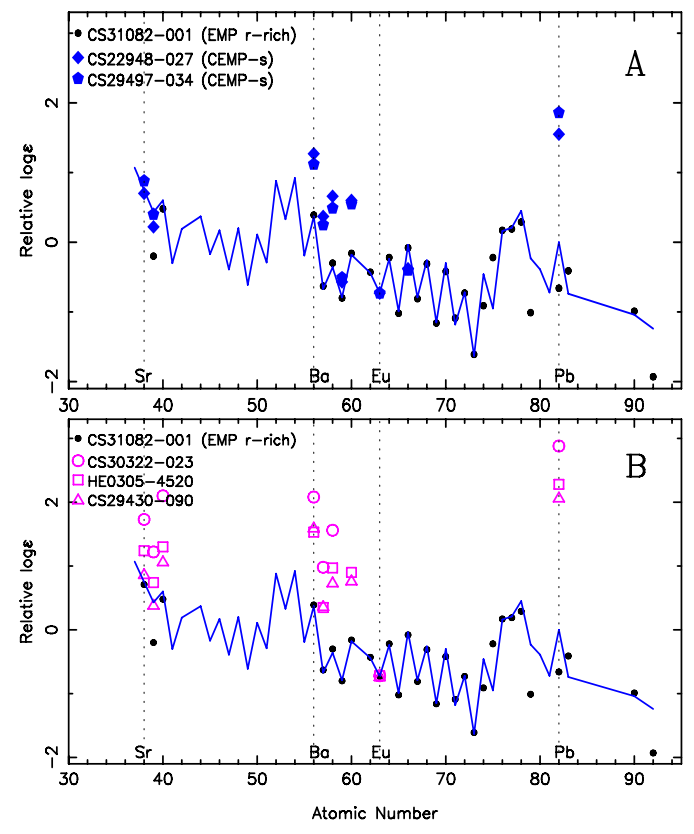

Fig. 9. Panel A): abundance pattern of the heavy elements in two classical CEMP-s stars CS 22948-027 and CS 29497-034 (blue diamonds and blue pentagons) and compared to the abundance pattern of the classical r-rich EMP star CS 31082-001 (black dots). The blue solid line represents the abundance pattern of the r-process elements in the solar system. Panel B): same as Panel A), but for the three low-Sr/Ba stars studied here (open pink symbols). In this figure, the abundances are represented not by ratios compared to the Sun (as in the previous figures), but by the other classical notation $\log \epsilon$ : the logarithm of the number of atoms for $10^{12}$ atoms of $\mathrm{H}$, scaled to the abundance of europium. The abundance pattern of the three low Sr-Ba stars (Panel B)) present many similarities with the abundance pattern of CS 22948-027 and CS 29497034 (panel A)).

\section{- CS 29493-090}

On spectra obtained in 2003, Barklem et al. (2005) measured $R V=269.8 \mathrm{~km} \mathrm{~s}^{-1}$ in very good agreement with the value obtained from our 2005 spectra (Table 2).

- HE 0305-4520

On spectra obtained in 2002, Barklem et al. (2005) measured $R V=135.3 \mathrm{~km} \mathrm{~s}^{-1}$, in agreement with the value obtained from our 2006 spectrum (Table 2).

As a consequence, none of these low-Sr/Ba stars presents a clear variation in the radial velocity that could support the hypothesis that their atmosphere has accreted matter from an AGB companion. As noted by Masseron et al. (2006), however, "the absence of evidence is not evidence of absence". The stars could have a rather long period (although this is not favorable to a significant amount of pollution) or/and a small radial velocity amplitude. They could also have an orbit in a plane (nearly) perpendicular to the line of sight, and in this case their binarity could perhaps be detected from a variation in the astrometric position of the stars by the spatial mission Gaia (de Bruijne 2012).

\subsection{Is it possible that the low Sr/Ba stars are AGB stars ?}

If we exclude an external enrichment of the atmospheres of our low-Sr/Ba stars, we have to explore the possibility of an internal production of ${ }^{12} \mathrm{C},{ }^{13} \mathrm{C}, \mathrm{N}$, and the s-process elements with a transfer to the surface. In low-mass stars this is sometimes possible if the star is an AGB star.
In the sample of Aoki et al. (2013), among 260 stars only five stars present a low $\mathrm{Sr} / \mathrm{Ba}$ ratio. If a star stays one tenth of its life on the RGB (e.g. Iben 1991; Pietrinferni et al. 2004), then our stars should stay around 1.3 Gyrs on the RGB. Following Bloecker (1995) the lifetime of a star of about $1 M_{\odot}$, in the AGB phase, is only about $4.5 \times 10^{7}$ years. Therefore, we could expect to find about eight AGB stars in the Aoki et al. (2013) sample, a number compatible with the small number of low-Sr/Ba stars in the sample.

\subsubsection{Do we see an internal pollution of the atmosphere occurring in TP-AGB stars?}

In low-mass stars an internal production of ${ }^{12} \mathrm{C},{ }^{13} \mathrm{C}, \mathrm{N}$ and the s-process elements with a transfer to the surface occurs in thermally pulsating AGB (TP-AGB) star. During a thermal pulse (e.g. Käppeler et al. 2011), partial He burning occurs in the intershell, and after a limited number of thermal pulses, the convective envelope penetrates the top region of the He intershell and mixes newly synthesized material to the surface during the third dredge-up (see also: Campbell \& Lattanzio 2008; Campbell et al. 2010).

Masseron et al. (2006) suggests that CS 30322-023 could be a TP-AGB star, and this would explain the observed abundances by an internal (recent) enrichment in carbon, and in heavy elements bearing the signature of the s-process. This hypothesis is based on their having found that the gravity of CS 30322-023 is very low $(\log g=-0.3)$, and is reinforced by the fact that $\mathrm{H}_{\alpha}$ presents a variable emission, probably due to a stellar wind. However, Aoki et al. (2007) also studied this star and found a gravity $\log g=1$. They use a photometric temperature, while Masseron et al. (2006) determine the temperature by requiring that the abundance deduced from the Fe I lines be independent of the excitation potential of the line, including in their computations Fe I lines with very low excitation potentials, so sensitive to NLTE effects (Cayrel et al. 2004). This difference in temperature has no significant effect on the abundance ratios, but does induce a difference in gravity (since gravity is determined in both cases from the ionization balance between Fe I and Fe II). It will be very interesting to obtain a direct measurement of the luminosity (and thus of $\log g$ ) of this star from a future precise parallax provided by the spatial mission Gaia, defining the precise evolution status of CS 30322-023.

CS 29493-090 and HE 0305-4520 have similar abundance ratios as CS 30322-023, but they are hotter with a higher gravity $\left(\log g=1.3\right.$, see Table 3 ) and do not show emission in $\mathrm{H}_{\alpha}$. From Fig. 8 of Masseron et al. (2006), they cannot be TP-AGB, but they could be early-AGB. Could it be possible that, already at this phase of the stellar evolution, neutron-capture elements be produced in the stellar interior and brought to the surface by a deep mixing?

\subsection{Do we see the s-process production of the core He flash in the low-Sr/Ba stars?}

In metal-rich stars, during the helium flash at the tip of the RGB, convection cannot break the H-burning shell and bring the products of the helium-burning nucleosynthesis to the surface, since the active $\mathrm{H}$-burning shell provides an entropy barrier against mixing. However Campbell et al. (2010) have shown that in hyper metal poor stars $([\mathrm{Fe} / \mathrm{H}]<5.0)$, this barrier can be broken (helium flash induced mixing), and the material, processed in the helium burning shell, is carried to the stellar surface by 
a dredge-up event. Our low-Sr/Ba stars are not as metal-poor as the model computed by Campbell et al. (2010), but it would be interesting to check if in stars with $[\mathrm{Fe} / \mathrm{H}]=-3.0$ this process could sometimes not be, in some particular conditions, efficient.

\section{Conclusion}

We analyzed four extremely metal-poor stars (EMP) for which anomalously low-[Sr/Ba] ratios were reported in the literature (lower than the ratio of the typical main r-process). One of them (the only turnoff star) turns out to have a "normal" Sr/Ba ratio (and was discarded from this work). The three remaining stars are all cool evolved giants. The abundance ratios of the elements lighter than the iron peak are generally not compatible with the classical EMP stars (Figs. 3 to 6). Compared to the classical EMP stars these low-[Sr/Ba] stars present an enhancement of $\mathrm{C}+\mathrm{N}, \mathrm{Na}$, and $\mathrm{Mg}$. From their temperature and gravity they can be evolved RGB stars or AGB stars.

The moderately high $\mathrm{C} / \mathrm{Fe}$ ratio measured in the three giants does not make them CEMP stars following the definition of Beers \& Christlieb (2005), but we showed that these cool giants $\left(T_{\text {eff }} \leq 4800 \mathrm{~K}\right)$ with a low gravity $(\log g<1.5)$ present the characteristics of stars having undergone a deep mixing (in the RGB phase). In this case, part of their initial $\mathrm{C}$ would have been transformed into $\mathrm{N}$ during their evolution, therefore the carbon abundance in the cloud that formed the star was probably much higher than the value measured today in their atmosphere; also the stars could be CEMP stars (following the definition of Beers $\&$ Christlieb 2005) at their birth. For evolved giants, it would be more suitable to define the CEMP type from the $\mathrm{C}+\mathrm{N}$ abundance of the star rather than from their $\mathrm{C}$ abundance alone: for example, CEMP stars could be defined (Fig. 3 ) by $[(\mathrm{C}+\mathrm{N}) / \mathrm{Fe}]>0.8$.

In the CEMP-s stars, the low $\mathrm{Sr} / \mathrm{Ba}$ ratio is generally associated to $[\mathrm{Ba} / \mathrm{Fe}]>1$ (Fig. 7), and to high abundances of $\mathrm{Ba}$ and $\mathrm{Pb}$ relative to $\mathrm{Eu}$. The radial velocity of these stars is variable (Starkenburg et al. 2014), and their abundances are interpreted as a superficial pollution (by a mass transfer from a now extinct AGB companion) providing $\mathrm{C}, \mathrm{N}$, and the neutron-capture elements produced by the s-process.

The abundance pattern of the neutron-capture elements in our three low-Sr/Ba stars is slightly different from the pattern of CEMP-s stars: the abundances of the neutron-capture elements relative to europium is larger (Fig. 9). The distribution of the ratios $[X / \mathrm{Fe}]$ (where $X$ is a neutron-capture element) vs. the atomic number $Z$ is very similar to the predictions of Goriely \& Mowlavi (2000) or Campbell et al. (2010) for a pure s-process.

What is the origin of the enrichment by the s-process?

We consider different possibilities:

- The stars could be classical RGB stars that would have undergone an external enrichment by a now extinct AGB star, as stated for the CEMP-s stars. In this case, we should expect, at least statistically, a variation in the radial velocity of these stars. No variation is present clearly in any of our three low-Sr/Ba stars but a further monitoring of their radial velocity would be very useful for a firm decision.

- In the absence of radial velocity variations, it is interesting to investigate whether autopollution is a valuable alternative solution.

A He shell flash may be considered, but it would occur when the stars are already in the TP-AGB phase, and it cannot occur in an early-AGB. As a consequence, this process does not seem able to explain the peculiar abundances of the low-Sr/Ba stars, which have a relatively high gravity $(\log g \sim 1.3)$.

The stars may have undergone a dual core flash at the tip of the RGB, but this mechanism is predicted only in hyper low metallicity stars (Campbell \& Lattanzio 2008; Campbell et al. 2010) and a higher $\mathrm{C} / \mathrm{N}$ ratio would be expected. Moreover, the surface composition is expected to remain unchanged from the core flash dredge-up until the beginning of the TP-AGB phase.

More definitive conclusions would require both (i) a check of a possible binarity of the three low-Sr/Ba stars from new precise measurements of the radial velocities, and also of the astrometric position of the stars (space astrometry mission Gaia). (ii) Measurements of the luminosity (distance) of the stars with Gaia, to derive their evolution status more precisely: early-AGB or TP-AGB. Maybe in the future, asteroseismology could help to distinguish between RGB and AGB stars.

Acknowledgements. We made use of SIMBAD operated by the CDS in Strasbourg, of the ADS Service (SAO/NASA), and of the arXiv Server (Cornell University). The authors acknowledge the support of Observatoire de ParisGEPI and the support of the CNRS (PNCG and PNPS). E.C. is grateful to the FONDATION MERAC for funding her fellowship.

\section{References}

Alvarez, R., Plez, B. 1998, A\&A, 330, 1109

Andrievsky, S. M., Spite, M., Korotin, S. A., et al. 2007, A\&A, 464, 1081

Aoki, W., Ryan, S. G., Norris, J. E., et al. 2001, ApJ, 561, 346

Aoki, W., Ryan, S. G., Norris, J. E., et al. 2002, ApJ, 655, 492

Aoki, W., Beers, T. C., Christlieb, N., et al. 2007, ApJ, 580, 1149

Aoki, W., Suda, T., Boyd, R. N., Kajino, T., \& Famiano, M. A. 2013, ApJ, 766, 13

Barbuy, B., Spite, M., Spite, F., et al. 2005, A\&A, 429, 1031

Barklem, P. S., Christlieb, N., Beers, T. C., et al. 2005, A\&A, 439, 129

Beers, T. C., \& Christlieb, N. 2005, ARA\&A, 43, 531

Behara, N. T., Bonifacio, P., Ludwig, H.-G., et al. 2010, A\&A, 513, A72

Bisterzo, S., Gallino, R., Straniero, O., Cristallo, S., \& Käppeler, F. 2012, MNRAS, 422, 849

Bloecker, T. 1995, A\&A, 299, 755

Bonifacio, P., Spite, M., Cayrel, R., et al. 2009, A\&A, 501, 519

Caffau, E., Bonifacio, P., François, P., et al. 2011, Nature, 477, 67

Caffau, E., Bonifacio, P., François, P., et al. 2012, A\&A, 542, A51

Campbell, S. W., \& Lattanzio, J. C. 2008, A\&A, 490, 769

Campbell, S. W., Lugaro, M., Karakas, A. I. 2010, A\&A, 522, L6

Carollo, D., Freeman, K., Beers, T., et al. 2014, ApJ, 788, 180

Cayrel, R., Depagne, E., Spite, M., et al. 2004, A\&A, 4161117

de Bruijne, J. H. J. 2012, Ap\&SS, 341, 31

Dekker, H., D’Odorico, S., Kaufer, A., Delabre B., \& Kotzlowski, H. 2000, Proc. SPIE, 4008, 534

Depagne, E., Hill, V., Spite, M., et al. 2002, A\&A, 390, 187

François, P., Depagne, E., Hill, V., et al. 2007, A\&A, 476, 935

Frebel, A., Collet, R., Eriksson, K., Christlieb, N., \& Aoki, W. 2008, ApJ, 684, 588

Gehren, T., Liang, Y. C., Shi, J. R., Zhang, H. W., \& Zhao, G. 2004, A\&A, 413, 1045

Goriely, S., \& Mowlavi, N. 2000, A\&A, 362, 599

Gustafsson, B., Bell, R. A., Eriksson, K., \& Nordlund, A.. 1975, A\&A, 42, 407

Gustafsson, B., Edvardsson, B., Eriksson, K., et al. 2003, in Stellar Atmosphere Modeling, eds. I. Hubeny, D. Mihalas, \& K. Werner, ASP Conf. Ser., 288, 331

Hill, V., Plez, B., Cayrel, R., et al. 2002, A\&A, 387, 560

Honda, S., Aoki, W., Ishimaru, Y., Wanajo, S., \& Ryan, S. G., 2006, ApJ, 643, 1180

Honda, S., Aoki, W., Ishimaru, Y., \& Wanajo, S. 2007, ApJ., 666, 1189

Iben, I. Jr. 1976, ApJ 208, 165

Iben, I. Jr. 1991, ApJS 76, 55

Izzard, R. G., Glebbeek, E., Stancliffe, R. J., \& Pols, O. R. 2009, A\&A, 508, 1359

Käppeler, F., Gallino, R., Bisterzo, S., \& Aoki, W. 2011, Rev. Mod. Phys., 83, 157 
M. Spite: Sr poor extremely metal-poor stars

Langanke, K., \& Thielemann, F.-K., 2013, ENews 44, 24

Lucatello, S., Tsangarides, S., Beers, T. C., et al. 2005, ApJ, 625, 825

McWilliam, A., Preston, G. W., Sneden, C., \& Searle, L. 1995, AJ, 109, 2757

Mashonkina, L., \& Christlieb, N. 2014, A\&A, 565, A123

Mashonkina, L., \& Gehren, T. 2001, A\&A, 376, 323

Mashonkina, L., Christlieb, N., Barklem, P. S., et al. 2010, A\&A, 516, A46

Mashonkina, L., Ryabtsev, A., \& Frebel, A. 2012, A\&A, 540, A98

Masseron, T., Van Eck, S., Famaey, B., et al. 2006, A\&A, 455, 1059

Masseron, T., Johnson, J. A., Plez, B., et al. 2010, A\&A, 509, A93

Masseron, T., Johnson, J. A., Lucatello, S., et al. 2012, ApJ, 751, 14

Pietrinferni, A., Cassisi, S., Salaris, M., \& Castelli, F. 2004, ApJ, 612, 168

Plez, B. 2012, ascl.soft05004P, http://adsabs.harvard.edu/abs/ 2012ascl. softQ5004P

Plez, B., Hill, V., Cayrel, R., et al. 2004, A\&A, 428, L9

Preston, G. W., \& Sneden, C. S. 2000, AJ, 120, 1014
Roederer, I. U., Cowan, J. J., Karakas, A. I., et al. 2010, ApJ, 724, 975 Sbordone, L., Bonifacio, P., Caffau, E., et al. 2010, A\&A 522, A26 Siqueira Mello Jr, C., Spite, M., Barbuy, B., et al. 2013, A\&A, 550, A122

Sivarani, T., Bonifacio, P., Molaro, P., et al. 2004, A\&A, 413, 1073 Sivarani, T., Beers, T. C., Bonifacio, P., et al. 2006, A\&A, 459, 125 Sneden, C., McWilliam, A., Preston, G. W., et al. 1996, ApJ, 467, 819

Sneden, C., Cowan, J. J., Lawler, J. E., et al. 2003, ApJ, 591, 936 Spite, M., \& Spite, F. 2014, Astron. Nachr., 335, 1, 65

Spite, M., Cayrel, R., Plez, B., et al. 2005, A\&A, 430, 655

Spite, M., Cayrel, R., Hill, V., et al. 2006, A\&A, 455, 291

Spite, M., Caffau, E., Bonifacio, P., et al. 2013, A\&A, 552, A107

Starkenburg, E., Shetrone, M. D., McConnachie, A. W., \& Venn, K. A. 2014 MNRAS, 441, 1217

Suda, T., Katsuta, Y., Yamada, S., et al. 2008, PASJ, 60, 1159

Yong, D., Norris, J. E., Bessell, M. S., et al. 2013, ApJ, 762, 26 